\title{
Исследование теплообмена в неподвижном плотном слое гранулированного материала
}

\author{
А. В. Солодкая \\ Одесская национальная академия пищевых технологий, ул. Канатная, 112, Одесса, 65039, Украина \\ ORCID: http://orcid.org/0000-0002-4043-7667
}

\begin{abstract}
Представлены результаты экспериментального исследования нагрева неподвижного слоя керамзита при его контакте с движущейся воздушной средой. Получены характерные кривые изменения температур газового и твердого компонентов на входе и выходе из аппарата при различных массах загрузки и температурах воздуха на входе. Проведен анализ условий, влияющих на выбор раџиональной толщины слоя засыпки. Показано, что данные по теплоте нагрева материала для условий проведения опытов могут быть обобщены единой зависимостью.
\end{abstract}

Ключевые слова: Тепловой аккумулятор; Теплообмен; Плотный слой; Температурные кривые; Тепловой баланс

\section{Дослідження теплообміну в нерухомому шарі гранульованого матеріалу}

\section{А. В. Солодка}

Одеська національна академія харчових технологій, вул. Канатна, 112, Одеса, 65039, Україна

\begin{abstract}
Наведено результати експериментального дослідження нагріву нерухомого шару керамзиту при його контакті з рухомим повітряним середовищем. Отримано характерні криві зміни температур газового $і$ твердого компонентів на вході і виході з апарату при різних масах завантаження $і$ температурах повітря на вход Проведено аналіз умов, щуо впливають на вибір раціональної товщини шару засипки. Показано, щчо дані по теплоті нагріву матеріалу для умов проведення дослідів можуть бути узагальнені єдиною залежністю.
\end{abstract}

Ключові слова: Тепловий акумулятор; Теплообмін; Щільний шар; Температурні криві; Тепловий баланс

DOI: http://dx.doi.org/10.15673/ret.v53i4.704

(C) The Author(s) 2017. This article is an open access publication

This work is licensed under the Creative Commons Attribution 4.0 International License (CC BY)

http://creativecommons.org/licenses/by/4.0/

\section{1. Введение}

Перенос теплоты к частицам от потока газа, проходящего через слой дисперсного материала, определяет во многих случаях скорость и устойчивость процессов, протекающих между твердым и газовым компонентом $[1,2]$. Примерами таких процессов могут быть сжигание топлива в слое, причем в последнее время наибольший интерес представляет сжигание биомассы [3, 4], нагрев сыпучего материала [5], процессы гетерогенного катализа $[6,7,8]$ сушка в слое $[9,10,11,12]$. Область исследований данной работы непосредственно относится к оптимизации аккумуляторов теплоты регенеративного типа, в которых нагретый газ из вытяжных устройств, к примеру, различных пищевых производств, проходя через плотный слой дисперсного материала, отдает свою теплоту, которую затем предполагается применять в различных целях. Основным недостатком аккумуляторов с дисперсным (гранулированным) материалом считается их большая масса, однако на основе проведенных исследований [13] было обосновано применение керамзита, который отличается существенно более низкими (в сравнении с другими материалами) плотностью и стоимостью. В общем случае, при проектировании регенеративных систем необходимо располагать сведениями о влиянии на их работу ряда факторов, таких как: начальная температура, расход теплоносителей; длительность цикла; материал, форма и геометрические размеры насадки регенератора. Недостаточная изученность процессов теплообмена не позволяет определить рациональные режимные параметры работы теплообменных аппаратов регенеративного типа с неподвижной дисперсной насадкой и создать основы для конструирования эффективных теплообменников-аккумуляторов. Целью работы являлась оценка влияния определяющих величин, таких как входные температуры компонентов, длительность процесса, масса загрузки, а также геометрические характеристики аппарата на процесс теплообмена между потоком воздуха и плотного слоя дисперсной насадки. 


\section{2. Экспериментальная установка и методика проведения эксперимента}

Схема экспериментальной установки приведена на рисунке 1 .

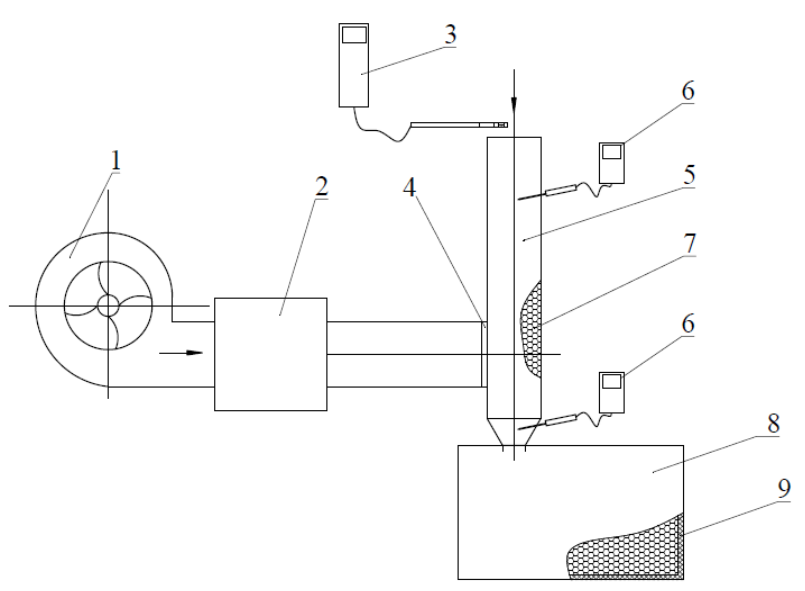

1 - вентилятор; 2 - нагреватель; 3 -анемометр; 4 сетка; 5 - рабочая камера;6 - термометр; 7 - дисперсный материал; 8 - емкость сбора дисперсного материала; 9 - изолячия

Рисунок 1 - Установка для экспериментального исследования теплообмена в плотном слое гранулированного материала

Методика проведения эксперимента заключалась в следующем. В рабочую камеру 5 загружался гранулированный материал. Включался вентилятор 1 и нагреватель 2, после которого нагретый воздух поступал в рабочую камеру. Температура воздуха контролировалась на входе и скорость потока, температура воздуха и материала на выходе (датчики 3 и 6). Эксперимент проводился с трехкратным повторением. Средняя скорость фильтрации воздуха составляла $w=0,5 . .2$ м/с, диаметр воздуховода $d=0,1 \mathrm{M}$.

Методика эксперимента заключалась в следующем. Устанавливалась заданная температура воздуха на регуляторе и включался вентилятор. Расход воздуха регулировался шибером на входе. После выхода установки в режим засыпался керамзит с заданной массой и начинали производиться замеры температуры. Измерялась температура воздуха и материала на входе и выходе установки, а также температура материала на расстоянии 0,12 м от уровня засыпки теплообменного участка. Максимальная высота слоя составляла 0,52 м., диаметр канала - 0,1 м. Замеры температур осуществлялись с интервалом времени 30 с. Температура воздуха на входе варьировалась от $60^{\circ} \mathrm{C}$ до $80^{\circ} \mathrm{C}$. По полученным результатам определялись основные характеристики теплообмена между воздухом и дисперсной насадкой.

\section{3. Результаты и обсуждение}

По полученным результатам анализировалось влияние длительности процесса, массы засыпки и входных температур на распределение температур твердого и газового компонентов, а также производились расчеты переданной теплоты.

На рисунке 2 представлен график зависимости температуры керамзита от времени при различном расстоянии от входа.

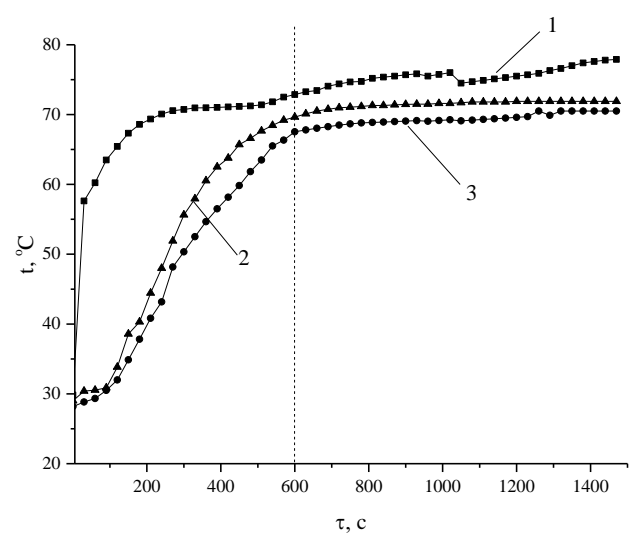

1 - температура материала на входе, 2 - температура материала на расстоянии 0,39 м от входа, 3 - температура на выходе 0,52 м.

Рисунок 2 - Изменение температуры керамзита на различном расстоянии от входа Температура воздуха на входе $t_{6}{ }^{\prime}=80 \mathrm{C}$

Как видно из графика, температура материала по высоте различается даже при наступлении стационарного режима, что связано с тепловыми потерями в окружающую среду. Материал на входе нагревается наиболее интенсивно. Температура материала на выходе не превышает $71 \mathrm{C}$, а интенсивный нагрев материала заканчивается после $600 \mathrm{c}$ (на графике обозначено пунктирной линией) при температуре $68^{\circ} \mathrm{C}$. Интенсивность нагрева материала во входном сечении снижается после 240 с работы.

На рисунке 3 представлены зависимости изменения температур компонентов от времени.

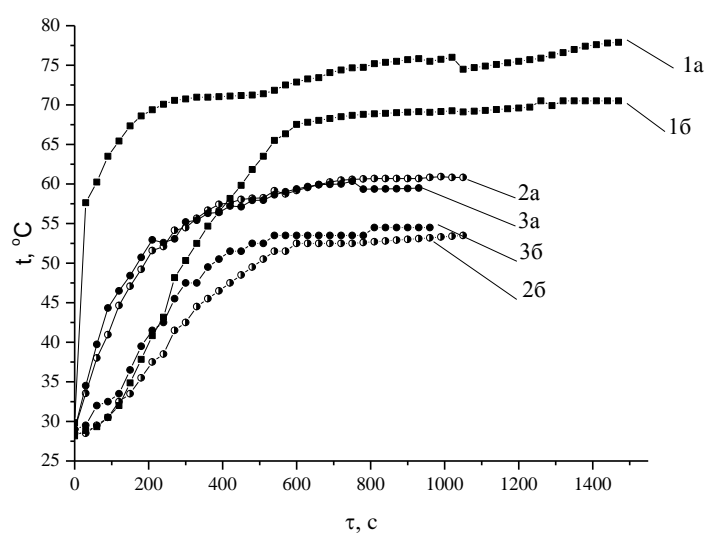

1 - температура воздуха на входе $t_{6}{ }^{\prime}=80 \mathrm{C}, 2,3$ - температура воздуха на входе $t_{6}{ }^{\prime}=60 \mathrm{C}, 1,2-$ масса загрузки $m=2,01 \kappa 2 ; 3$ - масса загрузки $m=1,7$ кг

Рисунок 3 - Изменение температуры керамзита в апnарате во входном, $x=0$ (a) и выходном $x=L$ (б) сечении annapama. 
Во всех опытах (см. рисунок 3) интенсивность нагрева материала во входном сечении существенно выше, чем на выходе. Уменьшение массы также приводит к увеличению интенсивности нагрева как во входном, так и выходном сечении. При $t_{\mathrm{B}}{ }^{\prime}=80^{\circ} \mathrm{C}$ темп нагрева в выходном сечении составляет $0,065 \mathrm{~K} / \mathrm{c}$, при $t_{\mathrm{B}}{ }^{\prime}=60^{\circ} \mathrm{C}$ темп нагрева в выходном сечении составляет $0,044 \mathrm{~K} / \mathrm{c}$ для массы загрузки $\mathrm{m}=2,01$ кг, а при $m=1,7$ кг темп нагрева составляет $0,059 \mathrm{~K} / \mathrm{c}$. При одинаковой массе загрузки (кривые 1,2 ) и одинаковой температуре нагрева, соответствующей температуре перелома в интенсивности для кривой $2, t=53^{\circ} \mathrm{C}$, темп нагрева для условий 1 составляет $0,1 \mathrm{~K} / \mathrm{c}$.

Отдельной важной задачей, возникающей при проектировании аккумулятора теплоты с дисперсной насадкой, является определение оптимальной толщины слоя. Рекомендация [15] относительно высоты слоя на основе гидродинамического критерия, согласно которой она должна составлять $h^{\prime}=(20 \ldots 30) d_{\ni}$, (где $d_{\ni}-$ эквивалентный диаметр частиц, для керамзита равный 0,025 м), не может быть принята. Как видно, для кривой 1 наблюдается существенное расслоение температур, при этом $h^{\prime}=0,52$ (это значение находится внутри указанного интервала). Для той же высоты засыпки, но при другой температуре воздуха на входе, температуры по высоте становятся практически одинаковыми. Это говорит о снижении вклада тепловых потерь от поверхности теплообменного участка в окружающую среду. При отсутствии тепловых потерь для неподвижного слоя можно рекомендовать выбор высоты слоя в зависимости от симплекса $\frac{t_{B}^{\prime}-t_{B}^{\prime \prime}}{t_{M}^{\prime \prime}-t_{\mathcal{M}}^{\prime}}$ и комплекса $\left(\frac{m \cdot c_{m}}{G_{B} c_{b} \tau_{\kappa}}\right)$, где $m$ - масса материала, $G_{b}-$ расход воздуха, $c_{b}-$ теплоемкость воздуха, $c_{m}$ - теплоемкость материала, $\tau_{\kappa}$ - длительность участка стабилизации. Анализируя полученные температурные кривые, можно отметить, что для условий проведения данных экспериментов можно принять $\tau_{\kappa}=690$ с. При других исходных данных это значение может быть определено по уравнению теплового баланса. Для получения рационального значения $h^{\prime}$ требуется проведение дополнительных экспериментов, однако можно проанализировать условия, которые способствуют оптимизации этого параметра. Рисунок 4 демонстрирует характер изменения температур материала и воздуха на входе и выходе аппарата для эксперимента с керамзитом при массе загрузки 1,7 кг. Видно, что с определенного момента времени (для условий данного эксперимента - начиная с 690 с) температура воздуха и материала в верхнем сечении аппарата. Это значение можно принять как окончание периода нагрева. Таким образом, рекомендуемое время нагрева - это время, при котором температура воздуха на выходе асимптотически приближается к температуре на входе (при отсутствии тепловых потерь), а температура материала становится практически равной температуре воздуха. Толщина слоя не может быть определена только из уравнения теплового баланса, т.к. следует также учитывать полноту омывания частиц потоком воздуха. Нагретый воздух по высоте аппарата должен эффективно взаимодействовать с поверхностью частиц, и в этом отношении увеличение высоты этому способствует. Прохождение газа через зернистый слой можно охарактеризовать как нерегулярное. Увеличение скорости газа способствует образованию отрывных течений, и при незначительной толщине слоя эффективность теплообмена будет снижаться.

Влияние высоты зернистого слоя $Н$ и скорости газового потока на газораспределение в зернистом слое описано в монографии [16]. Определено, что с увеличением $H$ наблюдается тенденция к снижению высоты пристенного всплеска скорости потока, а с ростом скорости наблюдается некоторое уменьшение отношения и сглаживание профиля скорости, это можно объяснить значительным увеличением потери напора в пристенной области по сравнению с остальной частью аппарата. В соответствии с [17] высота слоя, которую можно принять как высоту гидродинамической стабилизации, составляет $H_{\text {гидр.ст }}=(7-10) d_{3}$. Поэтому наименьшее значение высоты аппарата для насадки из керамзита составляет $H=(0,18-0,25)$ м. Однако этот критерий не может быть принят в качестве оптимального при выборе рекомендуемой высоты аппарата, как уже было показано, это значение зависит от входной температуры и скорости воздуха, а также начальной температуры материала. Для оценки оптимального значения высоты аппарата (слоя засыпки) следует рассчитать его высоту в соответствии с тепловым балансом:

$$
h^{\prime}=\left(\frac{4 \cdot G_{B} c_{B} \tau_{\kappa}}{\rho_{m} \cdot \pi \cdot D^{2} c_{m}}\right) \cdot \frac{t_{B}^{\prime}-t_{B}^{\prime \prime}}{t_{\mathcal{M}}^{\prime \prime}-t_{\mathcal{M}}^{\prime}},
$$

Затем, сравнив это значение с высотой гидродина-

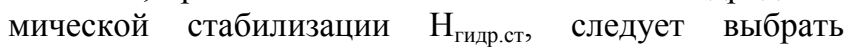
наибольшее.

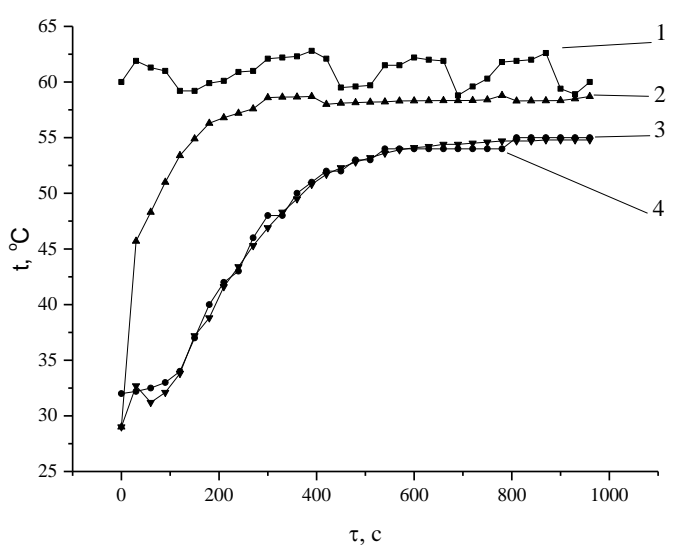

1 - температура воздуха на входе, 2 - температура материала на входе, 3 - температура воздуха на выходе, 4 - температура материала на входе (вверху аппаpama)

\section{Рисунок 4 - Температуры материала и воздуха на входе и выходе из аппарата.}

Температура воздуха на входе (кривая 1) изменялась во времени, что связано с регулировкой работы нагревательного устройства. Видно, что на выходе температура воздуха практически соответствует температуре материала, что свидетельствует о полноте поглощения 
теплоты передаваемой воздухом. Кривая 2 имеет сглаженный вид в сравнении с кривой 1, т.к. представляет собой результат усреднения данных по температуре для трех повторных экспериментов.

В выходном сечении наиболее явно прослеживаются три участка нагрева: начальный, непродолжительный, для которого температура изменяется незначительно, затем участок интенсивного нагрева с постоянной скоростью, и участок стабилизации, на котором температура материала практически не изменяется. Снижение массы способствует сближению температур воздуха на входе и материала.

Первичные данные экспериментов использовались для расчета теплоты, передаваемой от воздуха к материалу, и оценке коэффициента теплоотдачи. На рисунке 5 представлен характер изменения теплоты во времени для различных значений массы засыпки и температуры воздуха на входе. Анализ графика показывает, что в исследуемых условиях для всех случаев после 600 с нагрева поглощение теплоты из нагретого воздуха существенно снижается, т.е. его тепловой потенциал используется малоэффективно.

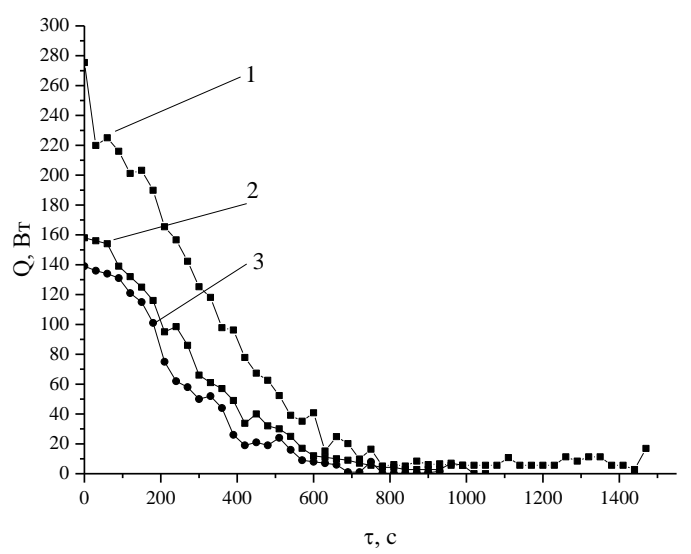

1 - температура воздуха на входе $t_{6}{ }^{\prime}=80^{\circ} \mathrm{C}, 2,3-$ температура воздуха на входе $t_{6}{ }^{\prime}=60^{\circ} \mathrm{C}, 1,3-$ масса загрузки $m=2,01 \kappa 2 ; 2-$ масса загрузки $m=1,7 \kappa 2$

\section{Рисунок 5 - Изменение теплоты нагрева материала} во времени.

Обобщающая зависимость для расчета теплового потока от воздуха к керамзиту имеет следующий вид:

$$
Q_{m}=Q_{80} \cdot\left(t_{B}^{\prime} / 80\right)^{1.76} \cdot(m / 2.1)^{1.23},
$$

где $Q_{80}=265,1-0,555 \cdot \tau+2,85 \cdot 10^{-4} \cdot \tau^{2}$ - теплота процесса при входной температуре воздуха $t_{B}^{\prime}=80^{\circ} \mathrm{C}$.

Сопоставительные расчеты теплового потока, передаваемого материалу, полученные из уравнения (2) и по уравнению теплового баланса $Q_{m}=Q_{b}-Q_{n o m}$, где $Q_{b}$ - теплота, переданная от воздуха по высоте канала и определяемая зависимостью $Q_{b}=G_{b} \cdot c_{b} \cdot\left(t_{b}^{\prime}-t_{b}^{\prime \prime}\right)$, $Q_{n o m}$ - потери в окружающую среду при естественной конвекции воздуха от наружной поверхности аппарата, определяемые в соответствии с зависимостями [14], показали расхождение результатов в пределах 7\%, что связано, очевидно, с погрешностью зависимости для расчета коэффициента теплоотдачи при естественной конвекции. Сравнительная оценка результатов расчета тепловых потоков позволяет сделать вывод о достоверности полученных при проведении экспериментов данных.

\section{Выводы}

Интенсивность нагрева материала зависит от температуры воздуха на входе, массы загрузки, расстояния от входа. Уменьшение массы на 20\% приводит к увеличению интенсивности нагрева как во входном, так и выходном сечении на 25\%. Длительность установления тепловой стабилизации в слое керамзита определяется входной температурой воздуха и массой засыпки и находится в пределах $600-800$ с. Темп нагрева керамзита лежит в пределах $0,1 \mathrm{~K} / \mathrm{c}-0,044 \mathrm{~K} / \mathrm{c}$.

Рациональная высота слоя засыпки определяется сопоставлением величин высоты гидродинамической стабилизации и высоты, определяемой уравнением теплового баланса.

Теплота нагрева для всех условий проведения экспериментов описывается подобными кривыми, которые обобщаются единой зависимостью с максимальной погрешностью $14 \%$.

\section{Литература}

1. Аэров М.Э., Тодес О.М., Наринский Д.А. Аппараты со стационарным зернистым слоем. Гидравлические и тепловые основы работы. - Л: Химия, 1979.-176 с.

2. Зырянова О.В. Теплоперенос в гетерогенных системах / Санкт-Петербургский горный университет. СПб, 2016. 38 c.

3. Vassilev SV, Baxter D, Vassileva CG An overview of the behaviour of biomass during combustion: Part I. Phasemineral transformations of organic and inorganic matter // Fuel 2013, Pages 391-449

4. Spliethoff H. Power generation from solid fuels. Springer, London, 2010, XXXVIII, 674 p

5. Бобков В.И. Проблема наискорейшего нагрева плотного слоя дисперсного материала. Научное обозрение, 2015. - с. 143-147

6. Brian Trewyn Heterogeneous Catalysis for Today's Challenges: Synthesis, Characterization and Applications Editor: book series: RSC Green Chemistry, 2015. - 138 p.

7. Бочкарев В.В. Теория химико-технологических процессов органического синтеза. Гетерофазные и гетерогенно-каталитические реакции. Том. Полит. Ун-т. Томск, 2015. - $118 \mathrm{c}$.

8. Верниковская Н. В.,. Малоземов Ю. В, Покровская С. А. /Каталитические процессы в реакторах с неподвижным слоем: Учебно-методическое пособие для компьютерного курса по инженерной химии каталитических процессов / Новосиб. гос. ун-т. Новосибирск, 2008. 68 с.

9. Sheiman V. A., Zelepuga A. S. . Kinetics of heating disperse materials during drying in a vibrofluidized layer // Journal of engineering physics 1969, Vol 17, Iss 4, pp 1201-1208

10. Журавлев, А. П. Технология сушки зерна в комбинированном слое // Хлебопродукты. - 2012. - № 7. C. $42-43$ 
11. Ndukwu M C. Effect of drying temperature and drying air velocity on the drying rate and drying constant of cocoa bean. // Agric. Eng. Int.: the CIGR Ejournal. 2009. Vol. XI., April

12. Raka Noveriyan Putra. Tri Ayodha Ajiwiguna. Influence of Air Temperature and Velocity for Drying Process // Procedia Engineering. 2017. - Vol. 170, - Pages 516-519].

13. Солодка А.В. /Изучение процессов теплопереноса в теплообменнике с гранулированной насадкой [текст] / А.В. Солодка // Збірник наукових праць. - Одеса, OHАХТ. 2017.

14. Исаченко В.П., Осипова В.А., Сукомел А.С.
Издание 4-е перераб. и дополненное. - М.: Энергоиздат, 1981. - $415 \mathrm{c}$.

15. Прутских Д. А. Гидродинамика и теплообмен в регенераторе с дисперсной насадкой / Автореферат дисс. на соискание уч. степени к. т. н. Специальность: 05.14.04- Промышленная теплоэнергетика. - Воронеж: 2009. $-22 \mathrm{c}$.

16. Пушнов А., Балтренас П., Каган А., Загорскис А. Аэродинамика воздухоочистных устройств с зернистым слоем: монография. Вильнюс: Техника, 2010. 348 с.

17. Попов, Е. К.. Исследование аэродинамических неоднородностей в реакторах с неподвижным слоем катализатора: Дис. канд. техн. наук. Ярославль. 1980. - 50 с.

Отримана в редакції 16.05.2017, прийнята до друку 06.06.2017

\title{
Investigation of Heat Exchange in a Fixed of Granular Material
}

\author{
A. V. Solodkava \\ Odessa National Academy of Food Technologies, 112 Kanatnaya St., Odessa, 65039, Ukraine \\ ORCID: http://orcid.org/0000-0002-4043-7667
}

\begin{abstract}
The results of an experimental study of heat exchange between an air flow moving in a vertical channel of circular cross section and a fixed disperse nozzle constituting a dense layer of solid particles are presented. Claydite is used as the material of the nozzle. The data on air and material temperatures at the inlet and outlet of the heat-exchange section of the experimental bench under various conditions of the heat exchange process are analyzed. The temperature curves for heated (expanded clay) and heating (air) heat carriers for various air temperatures at the inlet to the apparatus, masses of claydite loading, and the duration of heating in the apparatus are demonstrated. The results of the evaluation of the rate of heating of the material under various experimental conditions are presented. The conditions for choosing the rational height of the backfill layer in accordance with the thermophysical parameters of the process and recommendations for the height of hydrodynamic flow stabilization were estimated. It is shown that the data on the heat of heating of the material for the experimental conditions can be generalized by a single dependence. Expediency of conducted research is justified by the need to save energy resources, in particular, when heat of exhaust gases is utilized by heat exchangers-heat accumulators.
\end{abstract}

Keywords: Thermal battery; Heat exchange; Dense layer; Thermal curves

\section{References}

1. Aerov M.E., Todes O.M., Narinskiy D.A. Apparatyi so statsionarnyim zernistyim sloem. Gidravlicheskie i teplovyie osnovyi rabotyi. - L: Himiya, 1979.- 176 p.

2. Zyiryanova O.V. Teploperenos v geterogennyih sistemah / Sankt-Peterburgskiy gornyiy universitet. SPb, 2016. $38 \mathrm{p}$.

3. Vassilev S.V., Baxter D., Vassileva C.G. An overview of the behaviour of biomass during combustion: Part I. Phase-mineral transformations of organic and inorganic matter // Fuel 2013, Pages 391-449

4. Spliethoff H. Power generation from solid fuels. Springer, London, 2010, XXXVIII, 674 p

5. Bobkov V.I. Problema naiskoreyshego nagreva plotnogo sloya dispersnogo materiala. Nauchnoe obozrenie, 2015, 143-147

6. Brian Trewyn Heterogeneous Catalysis for Today's Challenges: Synthesis, Characterization and Applications Editor: book series: RSC Green Chemistry, 2015. - 138 r.

7. Bochkarev V.V. Teoriya himiko-tehnologicheskih protsessov organicheskogo sinteza. Geterofaznyie i geterogenno-kataliticheskie reaktsii. Tom. Polit. Un-t. Tomsk, 2015. - $118 \mathrm{p}$.

8. Vernikovskaya N.V., Malozemov U.V., Pokrovskaya S. A. Kataliticheskie protsessyi v reaktorah s nepodvijnyim sloem: Uchebno-metodicheskoe posobie dlya kompyuternogo kursa po injenernoy himii kataliticheskih protsessov / Novosib. gos. un-t. Novosibirsk, 2008. 68 p.

9. Sheiman V.A., Zelepuga A.S. Kinetics of heating disperse materials during drying in a vibrofluidized layer.
Journal of engineering physics 1969, Vol 17, Iss 4, pp 1201-1208

10. Juravlev, A.P. Tehnologiya sushki zerna v kombinirovannom sloe // Hleboproduktyi. 2012, 7, 42-43

11. Ndukwu M.C. Effect of drying temperature and drying air velocity on the drying rate and drying constant of cocoa bean. Agric. Eng. Int.: the CIGR Ejournal. 2009. V.XI. Apr. 12. Raka Noveriyan Putra, Tri Ayodha Ajiwiguna. Influence of Air Temperature and Velocity for Drying Process // Procedia Engineering. 2017. Vol. 170, 516-519.

13. Solodka A.V. Izuchenie protsessov teploperenosa $v$ teploobmennike s granulirovannoy nasadkoy [tekst] / A.V. Solodka // Zbirnik naukovih prats. - Odesa, ONAFT.

14. Isachenko V.P., Osipova V.A., Sukomel A.S. Izdanie 4-e pererab. i dopolnennoe. - M.: EHnergoizdat, 1981. $415 \mathrm{p}$.

15. Prutskih D.A. Gidrodinamika i teploobmen v regeneratore s dispersnoj nasadkoj / Avtoreferat diss. na soiskanie uch. stepeni k. t. n. Special'nost': 05.14.04- Promyshlennaya teploehnergetika. - Voronezh: 2009. - 22 p.

16. Pushnov A., Baltrenas P., Kagan A., Zagorskis A. Aehrodinamika vozduhoochistnyh ustrojstv s zernistym sloem: monografiya. Vil'nyus: Tekhnika, 2010. 348 p.

17. Popov, E.K. Issledovanie aehrodinamicheskih neodnorodnostej $\mathrm{v}$ reaktorah $\mathrm{s}$ nepodvizhnym sloem katalizatora: Dic. kand. tekhn. nauk. YAroslavl. 1980. - 50 p. 